\title{
Visualization of Human Inner Ear Anatomy with High-Resolution MR Imaging at 7T: Initial Clinical Assessment
}

\author{
M.A. van der Jagt, W.M. Brink, M.J. Versluis, S.C.A. Steens, J.J. Briaire, A.G. Webb, J.H.M. Frijns, and B.M. Verbist
}

\begin{abstract}
BACKGROUND AND PURPOSE: In many centers, MR imaging of the inner ear and auditory pathway performed on 1.5T or 3T systems is part of the preoperative work-up of cochlear implants. We investigated the applicability of clinical inner ear MR imaging at 7T and compared the visibility of inner ear structures and nerves within the internal auditory canal with images acquired at 3T.
\end{abstract}

MATERIALS AND METHODS: Thirteen patients with sensorineural hearing loss eligible for cochlear implantation underwent examinations on 3T and 7T scanners. Two experienced head and neck radiologists evaluated the 52 inner ear datasets. Twenty-four anatomic structures of the inner ear and 1 overall score for image quality were assessed by using a 4-point grading scale for the degree of visibility.

RESULTS: The visibility of 11 of the 24 anatomic structures was rated higher on the $7 T$ images. There was no significant difference in the visibility of 13 anatomic structures and the overall quality rating. A higher incidence of artifacts was observed in the 7T images.

CONCLUSIONS: The gain in SNR at 7T yielded a more detailed visualization of many anatomic structures, especially delicate ones, despite the challenges accompanying MR imaging at a high magnetic field.

ABBREVIATION: SNHL = sensorineural hearing loss

$\mathbf{P}$ atients with sensorineural hearing loss (SNHL) have malfunction of the inner ear, cochlear nerve, or central auditory pathway. Treatment consists of amplification of sound or, in case of severe-to-profound SNHL, direct electrical stimulation of the cochlear nerve by a cochlear implant. MR imaging of patients with SNHL focuses on the integrity of the auditory pathways from the cochlea to the auditory cortex in the brain. In particular, the fluid-filled spaces of the labyrinth and internal auditory canal and the cerebellopontine angle are of interest because the most com-

Received April 28, 2014; accepted after revision June 9.

From the Departments of Otorhinolaryngology (M.A.v.d.J., J.J.B., J.H.M.F.) and Radiology (W.M.B., M.J.V., A.G.W., B.M.V.), C.J. Gorter Center for High-Field MRI (W.M.B., M.J.V., A.G.W.), and Leiden Institute for Brain and Cognition (J.H.M.F.), Leiden University Medical Center, Leiden, the Netherlands; and Department of Radiology (S.C.A.S., B.M.V.), Radboud University Medical Center, Nijmegen, the Netherlands.

This work was supported by Advanced Bionics.

Paper previously presented as a poster at: Conference on Implantable Auditory Prostheses, July 14-19, 2013; Lake Tahoe, California; as an oral presentation in Dutch at: Annual Ear, Nose, and Throat meeting, November 15, 2013; Maastricht, the Netherlands.

Please address correspondence to Johan H.M. Frijns, MD, PhD, Department of Otorhinolaryngology, Leiden University Medical Center, PO Box 9600, 2300 RC Leiden, the Netherlands; e-mail: J.H.M.Frijns@lumc.nl

- Indicates open access to non-subscribers at www.ajnr.org

http://dx.doi.org/10.3174/ajnr.A4084 monly identified pathologies that cause SNHL are found in these regions. ${ }^{1-5}$

One clear trend in the development of MR imaging systems has been the drive to higher magnetic field strengths. ${ }^{6}$ For clinical inner ear scanning, MR imaging scanners with a magnetic field strength of $1.5 \mathrm{~T}$ or $3 \mathrm{~T}$ are routinely used. The relatively recent introduction of commercial 7T scanners potentially enables an increased SNR, resulting in more detailed imaging of anatomic structures. Concerning inner ear imaging, the visualization of delicate and small-sized inner ear structures might benefit from such high-resolution imaging. This may result in new opportunities for obtaining normative measurements and evaluating pathologic alterations within the inner ear or associated nerves. Such detailed anatomic depiction has caused particular interest for assessment of candidates for cochlear implants because it gives decisive information on implantation feasibility, possible surgical risks, and choice of implant device. As such, it would aid in patient-specific preoperative planning of cochlear implantation and could provide valuable information for individualized assessment of insertion.

Transition from a conventional 3T scanner to a stronger 7T scanner is challenging, however, due to technical complexities accompanying the higher magnetic field strength. ${ }^{6}$ One of these technical complexities is the increased inhomogeneity of the static 
Demographic details of studied patients $(N=17)$

\begin{tabular}{lc}
\hline Sex & No. \\
Male & 8 \\
Female & 9 \\
Pathologic imaging reporting & \\
Cochlea malformation & $1^{\mathrm{a}}$ \\
Hypoplasia acoustic nerve & $1^{\mathrm{a}}$ \\
Fenestrel otosclerosis & 1 \\
Labyrinthitis ossificans & 1 \\
None & 14 \\
Etiology & \\
Congenital & \\
$\quad$ Pendred syndrome & 1 \\
Of unknown origin & 5 \\
Acquired & \\
$\quad$ Sudden deafness & 2 \\
MIDD & 1 \\
Otosclerosis & 2 \\
$\quad$ Rubella infection & 2 \\
Unknown & 4 \\
Duration of deafness, years (mean) & 23.2 \\
\hline
\end{tabular}

Note:-MIDD indicates maternally inherited diabetes and deafness. a Same patient.

$\left(B_{0}\right)$ and radiofrequency $\left(B_{1}\right)$ fields, typically featuring areas of low $\mathrm{B}_{1}$ close to the temporal lobes. The $\mathrm{B}_{0}$ inhomogeneities are caused primarily by the susceptibility difference between inner ear fluids and the surrounding bone, and the $\mathrm{B}_{1}$ inhomogeneities, by the elliptic shape of the head. ${ }^{7}$ Both of these effects can result in loss of signal in the inner ear region as previously described by Takahara et $\mathrm{al}^{8}$ and van Egmond et al. ${ }^{9}$ Additionally, the specific absorption rate, for which regulatory safety limits are defined, scales approximately quadratic with field strength, ultimately limiting the imaging speed at high fields in vivo. Recently, we introduced geometrically tailored dielectric pads to locally tailor the $\mathrm{B}_{1}$ distribution. These improved contrast homogeneity and transmit efficiency in the region of the inner ear without increasing the specific absorption rate, which contributed to the development of a high-resolution imaging protocol at $7 \mathrm{~T} .^{10}$

The aim of this study was the following: 1) to investigate the feasibility of clinical inner ear imaging at 7T MR imaging, and 2) to compare the visibility of inner ear structures and nerves within the internal auditory canal with images acquired at $3 \mathrm{~T}$.

\section{MATERIALS AND METHODS \\ Patients}

This prospective study was approved by the hospital institutional review board (P07.096). Patients with SNHL, eligible for cochlear implantation and referred for 3T imaging between December 2012 and May 2013, were asked to participate in the study. Exclusion criteria were age younger than 18 years and contraindications for exposure to the magnetic field. Seventeen candidates for cochlear implantation were enrolled in the study, 9 women and 8 men between 27 and 78 years of age. Etiology and the duration of hearing loss are described in the Table. All patients underwent MR imaging at 3T as part of the standard work-up for cochlear implantation. After giving written informed consent, 16 patients underwent an examination at 7T; 1 patient was excluded due to an intracranial foreign body of unknown composition. Three other patients were excluded after the scanning procedure due to the following reasons: Scans of 2 patients were incomplete due to premature termination of the scanning procedure due to an unspecified technical defect, and the scanning procedure of 1 patient had to be aborted due to claustrophobia. After the procedure, patients were asked if they had dizziness because this is a frequently reported but temporary side effect of scanning at 7T.

\section{Imaging Technique}

All patients underwent examination on an Achieva or Ingenia 3T system (Philips Healthcare, Best, the Netherlands) as part of the standard preoperative work-up. The following scan parameters were used for the T2-weighted TSE sequence: FOV $=130 \times$ $130 \times 24 \mathrm{~mm}, 0.6-\mathrm{mm}^{3}$ isotropic voxels, TR/TE/TSE factor $=$ $2400 \mathrm{~ms} / 200 \mathrm{~ms} / 73$, and 80 sections, resulting in an acquisition time of approximately 6 minutes. In addition, all patients were scanned on an Achieva 7T system (Philips Healthcare) as described by Brink et al, ${ }^{10}$ by using a quadrature transmit and 32channel receive coil (NM008A-7P-012; Nova Medical, Wilmington, Massachusetts). To improve contrast homogeneity and transmit efficiency, we positioned 2 sex-specific high-permittivity pads containing a suspension of barium titanate and deuterated water next to both ears. ${ }^{10}$ High-resolution T2-weighted images were acquired by using the following parameters: FOV $=180 \times$ $180 \times 24 \mathrm{~mm}, 0.3-\mathrm{mm}^{3}$ isotropic voxels, TR/TE/TSE factor $=$ $3000 \mathrm{~ms} / 200 \mathrm{~ms} / 69$, tip angle $/$ refocusing angle $=90^{\circ} / 135^{\circ}$, parallel imaging reduction factor $=2.5 \times 1.5$, and 160 sections. These resulted in an acquisition time of approximately 10 minutes.

\section{Image Analysis}

The high-resolution T2-weighted images acquired at both 3T and 7T were transferred to an OsiriX DICOM viewer (http://www. osirix-viewer.com). ${ }^{11}$ The images were anonymized and presented in randomized order. Evaluation was performed by 2 head and neck radiologists with 5 and 13 years of experience, respectively. Twenty-four anatomic structures of the inner ear were assessed by using a 4-point grading scale for the degree of visibility for diagnostic evaluation: $1=$ not assessable, $2=$ poor, $3=$ adequate, $4=$ excellent. The structures selected were those most relevant for cochlear implantation. In addition, we designated an overall score for diagnostic image quality: $1=$ not diagnostic, $2=$ poor, 3 = adequate, $4=$ excellent. Both ears were evaluated separately. Subsequently, the scores of the 2 ears and 2 observers were averaged and normalized into a parameter between 0 and 1 . An overview of the anatomic structures and their difference in rating is shown in Fig 1. The numbers I, II, and III refer to the basal, mid-, and apical turns, respectively.

\section{Statistical Analysis}

Statistical analysis was performed by using SPSS (IBM, Armonk, New York). To study the influence of observed asymmetric signal intensity between the right and left inner ears on the $7 \mathrm{~T}$ images, we performed a linear mixed model. Statistical differences per anatomic structure between the $3 \mathrm{~T}$ and $7 \mathrm{~T}$ scanners were determined by using a Wilcoxon signed rank test. The interrater variability was determined by the Cohen $\kappa$ coefficient. All tests were 2-tailed, and $P<.05$ was considered a statistically significant difference.

AJNR Am J Neuroradiol 36:378-83 Feb 2015 www.ajnr.org 379 
Score differences 7 Tesla - 3 Tesla

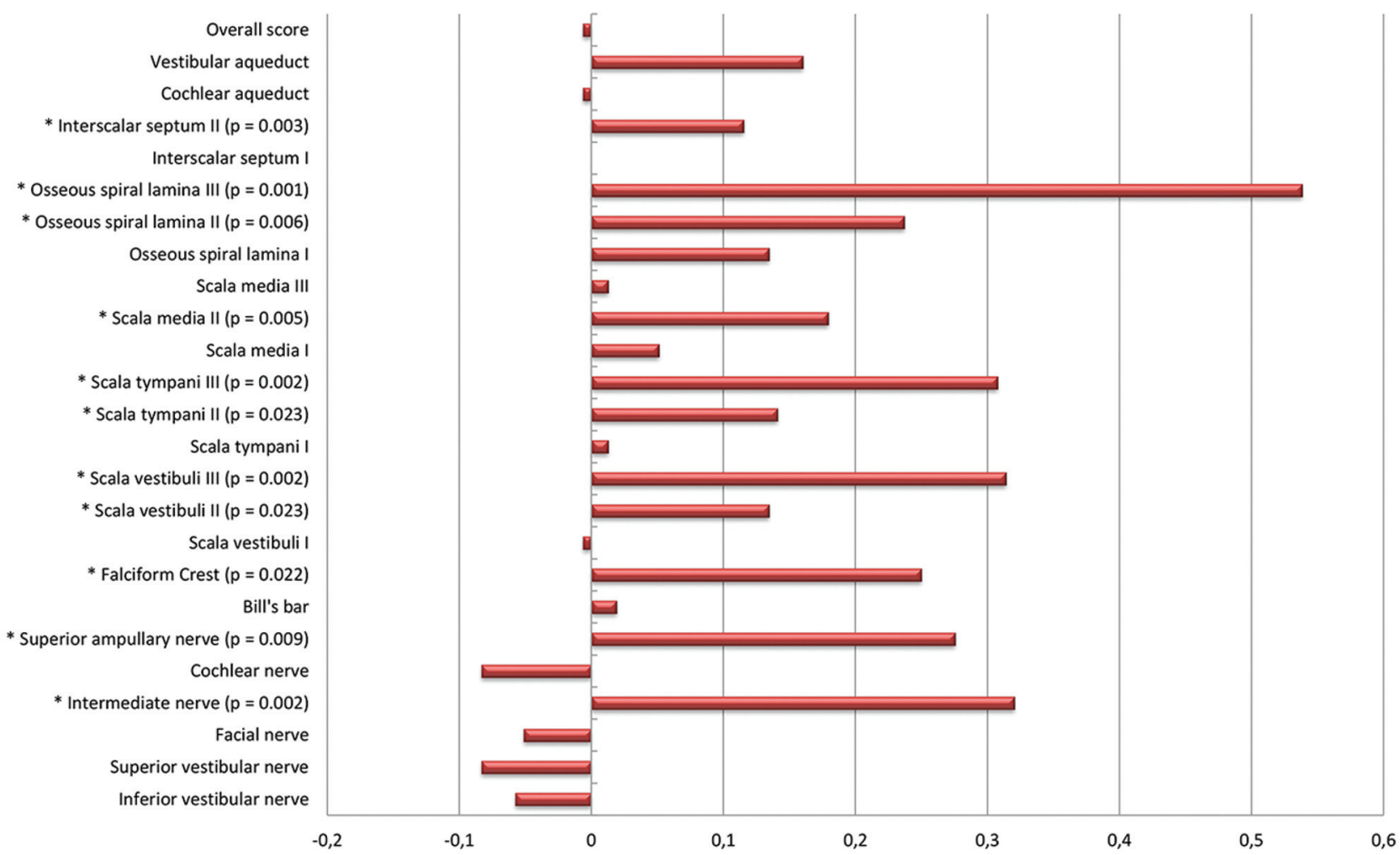

FIG 1. Mean differences in scoring of anatomic structures depicted at 3T and 7T. The bars on the right side of the zero line indicate differences in favor of the 7T images. The bars on the left side indicate differences in favor of the 3T images. The structures showing significant differences are marked with an asterisk on the left, and $P$ values are mentioned if significant.
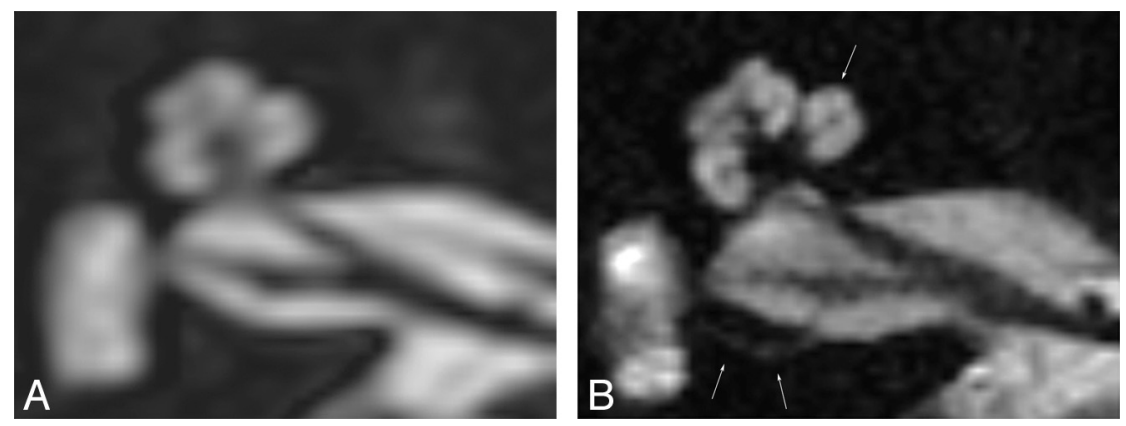

FIG 2. Axial cross-section of a right inner ear, rendered at $3 T(A)$ and 7T $(B)$; improved discrimination of the intracochlear structures and compartments is shown. In addition, sharper delineation of the nerves in the internal auditory canal is demonstrated. The single arrow indicates the scala media at the first turn. The double arrows indicate the superior ampullary nerve.

\section{RESULTS}

Twenty-six inner ears of 13 patients were available for image analysis. The occasionally observed asymmetric signal intensity on some of the 7T images did not result in a significantly different rating of the right and left inner ears $(P=.215)$. Therefore, no distinction between inner ear sides was used for analysis. Because 24 anatomic structures per inner ear were evaluated on T2weighted images acquired on $3 \mathrm{~T}$ and $7 \mathrm{~T}$ scanners plus an additional score for overall image quality, this process resulted in 2600 ratings applied by the 2 observers together. The ratings were averaged over ear and observer, leaving 650 ratings for statistical analysis. The visibility of 11 of the 24 anatomic structures was rated higher on the $7 \mathrm{~T}$ images. None of the anatomic structures were better depicted on the $3 \mathrm{~T}$ images. There was no significant difference in the visibility of 13 anatomic structures and the overall diagnostic image-quality rating. The interobserver agreement was moderate with a $\kappa$ value of 0.55 . None of the patients reported excessive or extended dizziness during or after the scan procedure.

\section{Cochlea}

Figure 2 shows the cochlea on an axial cross-section image, clearly illustrating the improved resolution of the $7 \mathrm{~T}$ image contributing to a more detailed depiction of the inner ear anatomy. Evaluated cochlear structures included the scala vestibuli, scala tympani, scala media, osseous spiral lamina, and interscalar septa. All structures were evaluated separately for each cochlear turn. Significant differences in favor of the 7T images were found for the scala tympani and vestibuli in the second and third turns, with a mean difference of $0.13(P=.023)$ and $0.31(P=.023)$ for the second turn and $0.14(P=.002)$ and 0.31 $(P=.002)$ for the third turn. The scala media in the first turn could be distinguished in 7 of 52 ratings on the $7 \mathrm{~T}$ images, but in none of the inner ears on the $3 \mathrm{~T}$ images. Visualization of the distinguished scala media was 6 times evaluated as "poor" and 1 

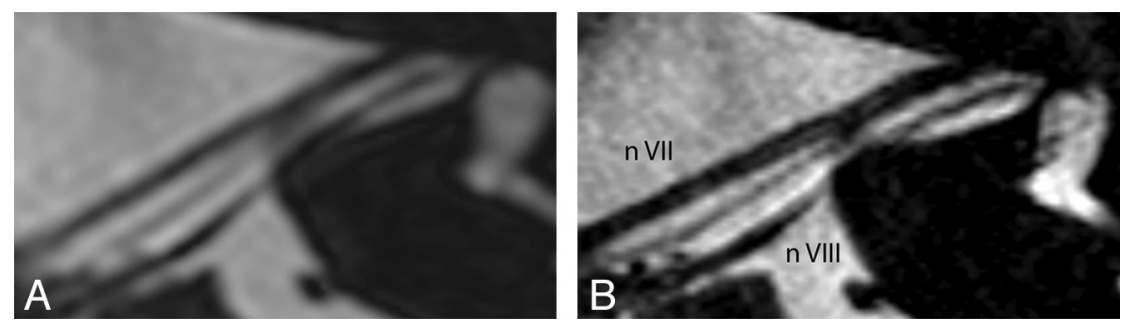

FIG 3. Axial cross-section along the course of the facial nerve of a left inner ear, rendered at 3T $(A)$ and 7T $(B)$. A sharp delineation of the neural structures and clear depiction of the intermediate nerve between cranial nerves VII and VIII are demonstrated on the 7T image.
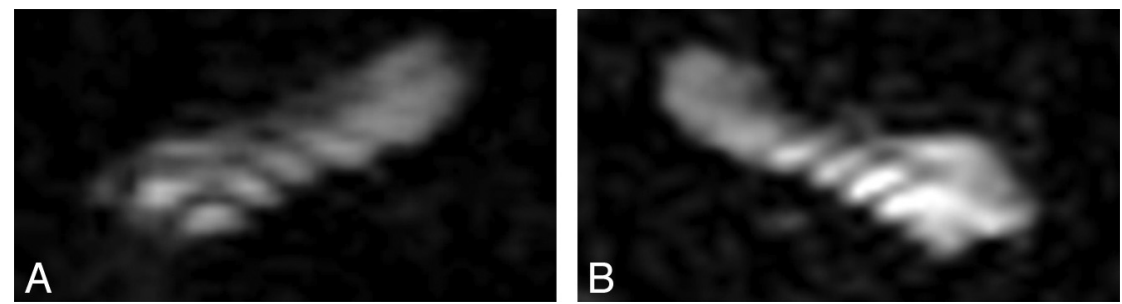

FIG 4. In 2 different patients, $7 T$ images showing stripelike-artifacts at the level of the first turn of right $(A)$ and left $(B)$ cochleas, disturbing the quality of the representation and impeding the distinction of the scala vestibuli and tympani.

time as "adequate." The resulting score difference of 0.05 was not significant $(P=.066)$. In the second turn, the scala media was visible in 21 inner ears on the 7T images, compared with none on the 3T images. The degree of visibility of these structures was rated "poor" in 16 cases, "adequate" in 3 cases, and "very good" in 2 cases. The score difference of 0.18 was significant for this turn $(P=.005)$. In the second and third turns, the depiction of the osseous spiral lamina was better on the $7 \mathrm{~T}$ images, resulting in a sharp delineation of the scala tympani and vestibuli $(P=.006$ for the second turn and $P=.001$ for the third turn). The visibility of the interscalar septum between the second and third turns also significantly benefited from high-resolution imaging at 7T $(P=.003)$.

\section{Internal Auditory Canal}

Statistical differences in visualization of the facial $(P=.259)$, superior $(P=.131)$, inferior vestibular $(P=.242)$, and cochlear nerves $(P=.151)$ through the internal auditory canal could not be demonstrated. On the $3 \mathrm{~T}$ images, the intermediate nerve was observed 5 of 52 times, compared with 31 times on the 7T images. On the $7 \mathrm{~T}$ images, the visibility of the intermediate nerve was evaluated as "poor" in 14 cases, "adequate" in 8 cases, and "excellent" in 9 cases. This evaluation resulted in a significant difference of $0.32(P=.002)$. An example of the clear depiction of an intermediate nerve is shown in Fig 3. In addition, a sharper delineation of the other neural structures was obtained. The superior ampullary nerve is indicated in Fig $2 B$ by 2 white arrows. This small neural structure is not regularly visualized on $3 \mathrm{~T}$ scans. In this study, it was observed 10 of 52 times on the 3T scans, 7 times as "poor" and 3 times as "adequate," compared with 28 times on the 7 T scans, 11 times as "poor," 6 times as "adequate," and 11 times as "excellent." These findings resulted in a significant difference of $0.28(P=.009)$.

The visualization of the falciform crest was significantly improved on the $7 \mathrm{~T}$ images; it was identified in 47 of 52 read- ings on the $7 \mathrm{~T}$ images, compared with 41 readings on the $3 \mathrm{~T}$ images. This finding led to a score difference of $0.25(P=$ .022). Bill's bar was only occasionally observed at either magnetic field strength.

\section{Cochlear and Vestibular Aqueducts}

Visualization of the vestibular and cochlear aqueducts did not differ significantly among the 3T and 7T images. A score difference of $0.16(P=.107)$ of the vestibular aqueduct and $0.01(P=.836)$ of the cochlear aqueduct was found.

\section{Artifacts}

A higher incidence of image artifacts was observed on the 7T images: 9 of the 13 scans versus none of the $3 \mathrm{~T}$ scans. These artifacts included motion artifacts likely due to the prolonged scan duration compared with 3T. Also, off-resonance effects due to the increased B0 inhomogeneities causing signal loss, and stripelike artifacts likely due to $B_{1}$ inhomogeneities were observed. An example of their appearances is shown in Fig 4.

\section{Overall Image Quality}

Image quality can be expressed as either the mean of scores per magnetic field strength or the actual applied score for image quality. First, we calculated the sum of scores separately for each magnetic field strength. Comparison of these values resulted in a significant difference of 0.11 per anatomic structure in favor of the 7T scanner $(P<.001)$. Second, the score for overall image quality as rated directly was analyzed. This score for overall image quality was applied in the context of diagnostic value, meaning distortion of the image quality by artifacts was taken into account. Comparing these scores did not show a significant difference between the 2 field strengths $(P=.631)$.

An overview of all the described outcomes is presented in Fig 1.

\section{DISCUSSION}

In patients with profound SNHL eligible for cochlear implantation, 7T MR imaging of the inner ear was successfully performed. Comparison with $3 \mathrm{~T}$ images demonstrated improved visualization of a large number of anatomic structures of the inner ear and internal auditory canal with high-resolution 7T imaging and emphasized the potential of clinical imaging at 7T.

Regarding the cochlear structures, the benefit of increased SNR was most pronounced for visualization of the microstructures of the second and third turns. The accurate distinction of the different turns and compartments is essential to accurately diagnose and localize pathologies and support surgical planning. One specific development during the past years that has emphasized the role of radiologic evaluation of cochlear implant candidates is the expanded criteria for cochlear implant recipients. A malformed cochlea is no longer an absolute contraindication for im- 
plantation; this change is important because as many as $20 \%$ of the patients with SNHL show some degree of inner ear malformation. ${ }^{12}$ However, when a malformation is present, the surgical procedure carries a higher risk for complications such as CSF gusher, and often a different surgical approach and electrode type must be chosen to ensure a good outcome. ${ }^{13}$ These considerations require precise preoperative planning; an increase in anatomic information as achieved with $7 \mathrm{~T}$ could be beneficial in such patients.

Another example in which an increase in anatomic information could be extremely relevant includes patients with obliterated cochleas. This fibrotic or osseous obliteration of the cochlear lumen is usually caused by meningitis-induced labyrinthitis. When parts of the cochlea are not patent, a different surgical approach should be followed with, in some cases, the use of a split array electrode. ${ }^{14}$ This device was developed to maximize the coverage of spiral ganglion cells by inserting 2 separate arrays through different cochleostomies. To precisely guide this procedure, comprehensive details of the cochlea anatomy are required. For electroacoustic stimulation, cochlear trauma needs to be minimized and preoperative delineation of the cochlear anatomy should be as accurate as possible. In addition, a gain in detailed anatomic information of the cochlea enables further research on morphologic characteristics, their influence on electrode position, and the relation of this position to the cochlear implant recipient's performance. ${ }^{15,16}$

At the internal auditory canal, smaller nerve branches such as the superior ampullary nerve and the intermediate nerve were, in general, better depicted at 7T. The fact that larger neural structures did not benefit from the increased resolution at 7T can be explained by motion artifacts, off-resonance and stripelike artifacts, and the scoring system. The internal auditory canal where these structures are housed was particularly vulnerable to patientinduced motion artifacts. It was observed that the neural structures in the internal auditory canal were more frequently affected than the cochlear structures when motion of the head occurred during the scan procedure. An explanation for this observation is not well-defined yet, but one can realistically hypothesize a combination of the direction of the motion and the dimensions of the internal auditory canal that makes image quality more vulnerable. Scanner-related artifacts such as the stripelike artifacts and offresonance were only pronounced at the higher field strength, as might be expected from the implicit larger absolute change in resonance frequency. Another contributing factor might have been the chosen 4 -point grading scale. In most cases, the visibility of nerves was rated "excellent" on the basis of delineation of the neural structures in both the $3 \mathrm{~T}$ and $7 \mathrm{~T}$ images. Consequently, a distinction in visualization between the 2 scanners was then hardly detectable and the presence of artifacts became decisive. Yet, although not evaluated systematically, one observer reported more confident assessment on cochlear nerve hypoplasia at 7T.

With respect to the clinical relevance, many etiologies causing SNHL cannot be seen in vivo with current techniques. Increasing the SNR and resolution, however, may demonstrate more anatomic changes related to SNHL. Showing the capability of 7T MR imaging to visualize anatomic structures such as the distinguished scalas of the second and third turn, scala media, intermediate nerve, and superior vestibular nerve is a first step toward that expectation. When etiologies are known, treatment and prognosis can be tailored more accurately.

Improved image quality does however, come with a number of drawbacks and limitations. An example of such a limitation is the prolonged scan duration. In our study, scan duration was prolonged from 6 to 10 minutes. This prolongation together with the lack of communication possibilities for this specific patient population caused an increased susceptibility to subject-induced artifacts; therefore, the use of communicative visual signaling during scanning is recommended. Additionally, the likelihood of motion artifacts could be reduced by shortening the scan duration through reduced-FOV imaging techniques. ${ }^{17}$ Another important issue is the presence of possible side effects during $7 \mathrm{~T}$ examinations. Previous research reported a slightly higher incidence of dizziness than at $3 \mathrm{~T}$, discomfort from the gradient noise, and a metallic taste. ${ }^{18-20}$ Nevertheless, these side effects are widely accepted, and in general, 7T examinations are well-tolerated. In our study population, none of the patients mentioned excessive discomfort during the scan procedure.

Another limitation of our study is the difference in background of the observers. One observer normally evaluates MR images acquired at $3 \mathrm{~T}$, whereas the second observer normally evaluates MR images acquired at $1.5 \mathrm{~T}$ only. This difference may have resulted in overvaluation of the $3 \mathrm{~T}$ images by the second observer, thereby diminishing the difference between the $3 \mathrm{~T}$ and $7 \mathrm{~T}$ images and decreasing the $\kappa$ value.

\section{CONCLUSIONS}

We report progress toward the use of 7T MR imaging for inner ear scanning in a clinical setting. The gain in SNR resulted in a more detailed visualization of a large number of relevant anatomic structures despite the remaining difficulties accompanying highmagnetic-field imaging. The findings of this study are encouraging for continued research on technical adjustments to push the limits of 7T MR imaging to reach its full potential and make it suitable for clinical applications.

Disclosures: Annerie van der Jagt—RELATED: Grant: Advanced Bionics, Comments: Advanced Bionics financially supports my PhD trajectory; Support for Travel to Meetings for the Study or Other Purposes: Advanced Bionics, Comments: Advanced Bionics paid for the expenses of attending the Conference of Implantable Auditory Prostheses in 2013 at Lake Tahoe, California, where these data were presented. Wyger M. Brink-RELATED: Grant: Nederlandse Organisatie voor Wetenschappelijk Onderzoek (No. 176.010.2005.030; A.G. Webb).* Jeroen J. Briaire-RELATED: Grant: Advanced Bionics, ${ }^{*}$ Comments: continuing-research grant for cochlear implant research; Support for Travel to Meetings for the Study or Other Purposes: Advanced Bionics, ${ }^{*}$ Comments: travel to a conference; Grants/Grants Pending: Advanced Bionics,* Comments: grant on cochlear implant research. Andrew G. Webb_RELATED: Grant: Organisation for Scientific Research Netherlands, ${ }^{*}$ Comments: funded project to develop applications for high-permittivity materials. Johan H. M. Frijns—RELATED: Grant: Advanced Bionics, ${ }^{*}$ Comments: a research grant paying the salary of M.A.v.d. Jagt; UNRELATED: Board Membership: Advanced Bionics, Comments: Medical Advisory Board; Consultancy: Advanced Bionics; Expert Testimony: as an ear, nose, and throat surgeon in the LUMC*; Grants/Grants Pending: Advanced Bionics, ${ }^{\star}$ STW, ${ }^{*}$ MED-El, ${ }^{*}$ ZonMW, ${ }^{*}$ Heinsius-Houbolt Fund, ${ }^{*}$ Comments: research grants in the field of cochlear implants; Royalties: STW, ${ }^{*}$ Comments: from my predecessor's work on middle ear implants. Berit M. Verbist-RELATED: Other: Advanced Bionics, ${ }^{*}$ Comments: financial support for PhD (Dr van der Jagt); UNRELATED: Payment for Lectures (including service on Speakers Bureaus): Bayer, ESMRMB, Comments: lectures/workshops on diagnostic imaging in the field of head and neck radiology; Royalties: LWW, Comments: coauthor of 2 books on head and neck radiology. *Money paid to the institution. 


\section{REFERENCES}

1. Verbist BM. Imaging of sensorineural hearing loss: a pattern-based approach to diseases of the inner ear and cerebellopontine angle. Insights Imaging 2012;3:139-53

2. Casselman JW. Diagnostic imaging in clinical neuro-otology. Curr Opin Neurol 2002;15:23-30

3. St Martin MB, Hirsch BE. Imaging of hearing loss. Otolaryngol Clin North Am 2008;41:157-78, vi-vii

4. Mohan S, Hoeffner E, Bigelow DC, et al. Applications of magnetic resonance imaging in adult temporal bone disorders. Magn Reson Imaging Clin N Am 2012;20:545-72

5. Davidson HC. Imaging evaluation of sensorineural hearing loss. Semin Ultrasound CT MR 2001;22:229-49

6. Robitaille P, Berliner L. Ultra High Field Magnetic Resonance Imaging (Biological Magnetic Resonance), Vol. 26. Berlin: Springer; 2006

7. Sled JG, Pike GB. Standing-wave and RF penetration artifacts caused by elliptic geometry: an electrodynamic analysis of MRI. IEEE Trans Med Imaging 1998;17:653-62

8. Takahara T, Hoogduin H, Visser F, et al. Imaging of the inner ear at 7T: initial results. In: Proceedings of the Annual Meeting of International Society of Magnetic Resonance in Medicine, Stockholm, Sweden. May 1-7, 2010:4448. Vol 17

9. van Egmond SL, Visser F, Pameijer FA, et al Ex vivo and in vivo imaging of the inner ear at 7 Tesla MRI. Otol Neurotol 2014;35:725-29

10. Brink WM, van der Jagt AM, Versluis MJ, et al. High permittivity dielectric pads improve high spatial resolution magnetic resonance imaging of the inner ear at 7 T. Invest Radiol 2014;49:271-77
11. Rosset A, Spadola L, Ratib O. OsiriX: an open-source software for navigating in multidimensional DICOM images. J Digit Imaging 2004;17:205-16

12. Sennaroglu L. Cochlear implantation in inner ear malformations: a review article. Cochlear Implants Int 2010;11:4-41

13. Sennaroglu L, Sarac S, Ergin T. Surgical results of cochlear implantation in malformed cochlea. Otol Neurotol 2006;27:615-23

14. Millar DA, Hillman TA, Shelton C. Implantation of the ossified cochlea: management with the split electrode array. Laryngoscope 2005;115:2155-60

15. van der Marel KS, Briaire JJ, Wolterbeek R, et al. Diversity in cochlear morphology and its influence on cochlear implant electrode position. Ear Hear 2014;35:e9-20

16. Holden LK, Finley CC, Firszt JB, et al. Factors affecting open-set word recognition in adults with cochlear implants. Ear Hear 2013;34:342-60

17. Wargo CJ, Moore J, Gore JC. A comparison and evaluation of reduced-FOV methods for multi-slice 7T human imaging. Magn Reson Imaging 2013;31:1349-59

18. Heilmaier C, Theysohn JM, Maderwald S, et al. A large-scale study on subjective perception of discomfort during 7 and $1.5 \mathrm{~T}$ MRI examinations. Bioelectromagnetics 2011;32:610-19

19. Theysohn JM, Maderwald S, Kraff O, et al. Subjective acceptance of 7 Tesla MRI for human imaging. MAGMA 2008;21:63-72

20. Versluis MJ, Teeuwisse WM, Kan HE, et al. Subject tolerance of $7 \mathrm{~T}$ MRI examinations. J Magn Reson Imaging 2013;38:722-25 